\title{
Cannabinoids and Sleep-Molecular, Functional and Clinical Aspects(2021) editors Monti JM, Pandi-Peumal SR, Murillo-Rodriguez E pp. 181 ISBN: 9,783,030,616,632 Springer
}

\author{
Arn H. Eliasson ${ }^{1}$ (1) \\ Received: 6 May 2021 / Revised: 6 May 2021 / Accepted: 15 May 2021 / Published online: 21 May 2021 \\ (c) The Author(s), under exclusive licence to Springer Nature Switzerland AG 2021
}

\begin{abstract}
In 13 chapters, the book approaches the topic of cannabinoids and sleep in an organized and thorough manner, providing clinicians and researchers with detailed descriptions of neurobiology and pathophysiology. The book will serve as a useful reference resource, especially for researchers in the field.
\end{abstract}

Keywords Book review · Sleep · Cannabinoids · Tetrahydrocannabinol · Cannabidiol · Cannabigerol · Receptors · Pathophysiology $\cdot$ Neurobiology $\cdot$ Pharmacology

\section{Discussion}

Colorful quotes by neurologist and psychopharmacology researcher Dr. Ethan Russo, and astronomer, astrophysicist, and science communicator Dr. Carl Sagan, give a salubrious tone to the subsequent chapters. The book is an exposition on the endocannabinoid system-a central player in biological physiology. Chapters are authored by an international cast of contributors from nine different nations including Brazil, Canada, Chile, France, Germany, Mexico, Spain, Uruguay, and the USA.

The range of topics discussed in the 13 chapters is ambitious and covers descriptions of the chemical constituents and derivatives of Cannabis sativa, cannabinoid receptors in the brain, sleep-wake neurobiology, and the effect of cannabinoids on sleep-wake control including sleep research in laboratory animals. The early chapters are detailed and extensive. It is easy for a clinician to get lost in the weeds. There is also substantial overlap of information provided among the early chapters-not necessarily a bad thing. Chapter 5 on sleep-wake neurobiology is an excellent detailed review of neuronal subtypes, molecules, and networks that regulate sleep.

Arn H. Eliasson

aheliasson@aol.com

1 Department of Medicine, Uniformed Services University of the Health Sciences, Bethesda, MD, USA
A recurrent theme in each of the chapters of the book is that there is a paucity of research on sorting out the specific ingredients in the cannabinoid preparations, their biological activities, and their clinical utility. The book therefore serves as a teaser for readers who would like to know more research-derived knowledge as well as potential clinical applications of tetrahydrocannabinol (THC), cannabidiol (CBD), and cannabigerol (CBG).

A number of chapters do suffer from problems with grammar and language usage but these defects do not substantially detract from the overall value of the book as a reference resource for researchers in the field of cannabinoid research. There are particularly helpful figures describing effects of cannabinoids (chapter 8) and numerous very useful tables that outline the findings of prior research in great detail.

The final four chapters address cannabinoids in the setting of clinical conditions such as disturbed sleep, circadian rhythm, addictions, and restless legs syndrome. While the passages include handy summaries of the clinical conditions, they end with a disappointing lack of credible data on clinical applications of cannabinoids in those settings.

Publisher's note Springer Nature remains neutral with regard to jurisdictional claims in published maps and institutional affiliations. 